\title{
Las competencias TIC de los profesores de posgrado en la Universidad de La Sabana: la mirada de sus estudiantes
}

\author{
ICT skills of posgraduate teachers at University of the Savannah: a \\ students perspective
}

\author{
Mónica Marcela Sánchez Duarte \\ Universidad de La Sabana \\ monica.sanchez@unisabana.edu.co \\ Antoni Navío Gámez \\ Universidad Autónoma de Barcelona \\ antoni.navio@uab.cat
}

Recibido: 04/07/2018

Aceptado: 07/09/2018

Publicado: 21/12/2018

\section{RESUMEN}

El presente escrito, muestra los resultados de un estudio de caso realizado en la Universidad de La Sabana (Colombia), que se fijó como objetivo identificar aquellos recursos y herramientas que proveen las Tecnologías de la Información y Comunicación -TIC- y que son de mayor uso por los profesores, desde la voz de los estudiantes. Participan seis programas de maestría. con la participación de 245 estudiantes y 64 profesores. Fue un estudio de corte mixto, para el cual se diseñaron y aplicaron cuestionarios en línea y se hizo un análisis estadístico descriptivo para esta primera fase. Se encontró que los recursos de preferencia para los innovadores son las plataformas, los videos y multimedia, por lo cual conceden especial importancia a su creación y desarrollo. Emplean de manera frecuente las herramientas básicas como los foros y el almacenamiento en la nube junto con los procesadores de texto y hojas de cálculo, dejando de lado otros recursos y herramientas más especializadas. Los estudiantes identifican que en su desempeño los profesores privilegian factores como el cumplimiento, a la par que favorecen la autonomía y la comunicación. Con respecto a los estándares de la competencia digital revisados: Unesco, ISTE, Ministerio de educación y Reglamentación 35, los profesores en cuestión no alcanzan los máximos en su desarrollo y por el contrario se sitúan en los niveles más bajos de dicha competencia. Queda un gran cuestionamiento sobre los profesores y programas de posgrado en educación donde se desconoce la existencia de profesores innovadores que empleen las TIC.

\section{PALABRAS CLAVE}

Profesor; posgrado; TIC; práctica innovadora.

\section{ABSTRACT}

This paper shows the research results of a study case at the University of La Sabana (Colombia). The main objective was to identifiy identify those resources and tools of ICTs in six master programs, according to the students perspective. It was a mixed like study, that included the participation of 245 students and 64 teachers. Online questionnaires were designed and applied, and a descriptive statistical analysis was made for this first phase. The findings reveal that the most popular resources for innovators are platforms, videos and multimedia; as a consequence, innovative teachers grant special importance to their creation and 
development. Continuos use of basic tools, such as forums and cloud storage, along with word processors and spreadsheets becomes evident. In the other hand they leave aside other resources and more specialized tools like web quest, podcast or games. Students identify that their teachers privilege items like fulfillment, autonomy and communication. With respect of the digital standards and competence reviewed: Unesco, ISTE, MEN and Regulation 35, teachers do not reach the maximum level; conversely they are at the lowest levels. Emerge another question about teachers and education graduate programs, about existence of innovative teachers using ICT.

\section{KEYWORDS}

Teacher; postgraduate; ICT; innovative practice.

\section{CITA RECOMENDADA}

Sánchez, M.M. y Navío, A. (2018). Las competencias TIC de los profesores de posgrado en la Universidad de La Sabana: la mirada de sus estudiantes. RIITE. Revista Interuniversitaria de Investigación en Tecnología Educativa, 5, 84-101. Doi: http://dx.doi.org/10.6018/riite/2018/336511

\section{Principales aportaciones del artículo y futuras líneas de investigación:}

- Identifica los recursos TIC que los profesores de posgrado usan habitualmente y cuáles no y da cuenta de aquellos factores que los profesores privilegian en torno a su forma de relacionarse en y para la enseñanza.

- Futura línea: estado de la formación en competencias TIC en profesores de posgrado.

\section{INTRODUCCIÓN}

En Colombia, la población de profesionales con formación posgradual es inferior a la del resto del continente, razón de peso para que las políticas y proyectos de estado en los últimos años, hayan dedicado recursos y especial atención a la formación de magísteres y doctores para compensar ese déficit que según Roa y Pacheco (2014) se aproxima al $71 \%$. Es así, que desde el año 2013 la Alcaldía Mayor de Bogotá a través de la Secretaría de Educación del Distrito SED-, gestionó recursos por $\$ 35.000$ millones de pesos para financiar el $70 \%$ de la matrícula de profesorado en los niveles de formación básica y media que desearan avanzar en sus titulaciones (Ministerio de Educación Nacional, 2013).

Para la escogencia de los programas e instituciones ofertantes se establecieron entre otros, los siguientes criterios: ser programas de excelencia, responder a las necesidades de sus profesores, incentivar la investigación e innovación educativa y, fortalecer la respectiva comunidad académica; además de manera colateral, lograr una trasformación pedagógica de la escuela, de la mano del desarrollo de proyectos de innovación. Las universidades participantes para este proyecto fueron aquellas con programas del área de la educación y con la acreditación de alta calidad otorgada por el Consejo Nacional de Acreditación - CNA-.

Los programas de formación posgradual para esta población de maestros en ejercicio deben responder con contenidos vigentes y una planta profesoral actualizada que conduzca a cumplir los propósitos trazados, conviniendo con Sánchez, Boix y Jurado (2009), cuando admiten que ser docente hoy, demanda nuevas formas de actuación, incluidas la actualización de conocimientos de la mano de la incorporación y empleo de las TIC para optimizar el trabajo. Este es un reto que enfrentan los profesores de posgrado, por cuanto se privilegia su experticia disciplinar por encima de las competencias pedagógica o tecnológica.

Es importante considerar, que el estudio se da en un contexto institucional que le apuesta a promover la competencia digital de su personal docente como un atributo fundamental para su vinculación y permanencia. Se presume que contar con docentes que tienen desarrollada su competencia digital, conducirá a que los estudiantes a su cargo, también desplieguen habilidades en su uso; así, impactarán su respectivo quehacer y sus ámbitos de trabajo para responder a la 
calidad que debe caracterizar los programas de una institución acreditada, como lo propone el CNA (CNA, 2010).

En congruencia, se ha considerado importante revisar cómo los profesores integran las TIC en su práctica de aula en 6 programas de maestría circunscritos al área de educación, identificando aquellos recursos y herramientas digitales de mayor uso, desde la voz de los estudiantes. El presente artículo se constituye en un avance del proyecto de doctorado Caracterización de la práctica docente mediada por TIC del profesorado de maestría de la Universidad de La Sabana realizado en Chía, Colombia.

\section{ESTADO DEL CONOCIMIENTO}

\subsection{Definiendo las competencias TIC para el profesorado}

La Unesco (1998) exhortó al profesorado universitario a aprovechar las posibilidades que las Tecnologías de la Información y las Comunicaciones - TIC- brindaban al proceso educativo con su permanente desarrollo. Posteriormente Tünnermann y De Souza (2003) basados en esas premisas, plantearon entre otros asuntos la necesidad de redefinir las competencias profesionales de dicha población, enfrentada a asumir un nuevo papel ante el protagonismo que han cobrado los discentes en sus procesos de construcción de conocimiento.

Se trae a colación en primer lugar, comprender el significado de competencia tecnológica, partiendo de Prendes Espinosa y Porlán Gutiérrez (2013) quienes consideran posible homologar el término con los constructos: competencia TIC o competencia digital, según procede de autores como Le Boterf (2000), Olcott y Schmidt (2002), Cano (2005), Hanna (2002) y Monereo (2005).

Como definición del concepto, se acude a Suárez-Rodríguez, Almerich, Díaz-García, y Fernández-Piqueras (2012, p. 294), quienes consideran las competencias TIC para el profesorado, como "el conjunto de conocimientos y habilidades necesarios que se deben poseer para utilizar estas herramientas tecnológicas como unos recursos educativos más integrados en su práctica diaria". Las aportaciones de Smonaitiené y Greenrod (2009, citados en Indrašienè, Dromantienè, \& Bielskytè-simanavičienè, 2015), complementan que esa competencia tecnológica significa más que conocimientos de computación y estrategias de aplicación de software y, que incluir las TIC facilita el proceso de aprendizaje siempre y cuando se utilicen convenientemente por profesores calificados y en los ambientes adecuados.

\subsection{Los estándares TIC para el profesorado del Siglo XXI}

En 2008 como producto de la revisión global del sistema educativo, la Unesco publicó el Marco de competencias TIC para profesores, que ha sido punto de referencia para fijar políticas y directrices, que orienten la alfabetización digital del profesorado en todos los niveles de formación. Allí, se promulga que el profesor es responsable de adecuar un ambiente que brinde no solo las oportunidades, sino que facilite a los estudiantes el empleo de tecnologías para aprender y comunicarse (Unesco, 2008).

Ese Estándar de competencias en TIC para profesores, busca mejorar las prácticas docentes en todos los frentes, combinando las competencias tecnológicas con innovaciones en la pedagogía y el Currículo, para contribuir a mejorar la calidad del sistema educativo (Unesco, 2008). Proporciona también, lineamientos para el profesorado en lo que concierne a estructurar planes de formación y entrenamiento, en orden a habilitarlos para que asuman ese rol crucial como formadores de estudiantes tecnológicamente competentes. Carrera y Coiduras (2012) rescatan las afirmaciones del organismo en torno a la competencia digital del profesor, señalándolo como un gestor para la comprensión y aplicación de las tecnologías en contexto, junto a la generación de conocimiento e innovación.

La propuesta de la Unesco considera tres enfoques: i) las nociones básicas (alfabetización tecnológica); ii) la profundización del conocimiento y, iii) creación de conocimiento. Cada enfoque repercute para el cambio y mejora de la educación sobre 5 componentes de dicho sistema: currículo, pedagogía, uso de TIC, organización y administración y, el desarrollo profesional docente (Unesco, 2008). 
La figura 1 muestra el mencionado marco de competencias:

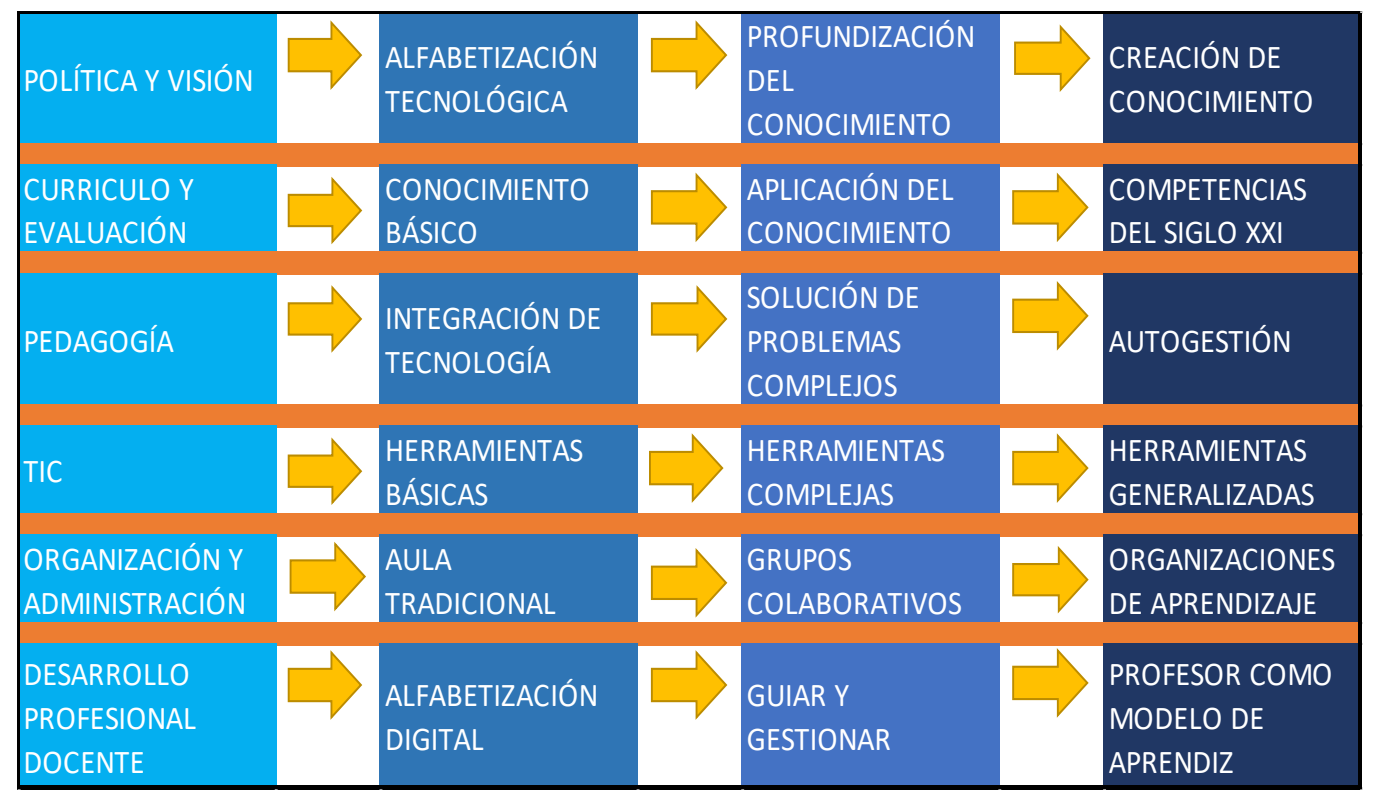

Figura 1. Marco de competencias TIC para profesores, Unesco (2008).

Otra autoridad en el tema es la International Society for Technology in Education (ISTE) que ha investigado y emitido varias publicaciones (2000, 2008 y 2018), consideradas por muchos autores como referente de las competencias digitales para docentes. Se ha difundido el reporte 2018 para educadores, con 7 estándares agrupados en dos grandes aspectos: el docente como profesional empoderado (Figura 2) y, como impulsor del aprendizaje (Figura 3). Cada uno de estos aspectos incluye unos roles con sus respectivos indicadores (ISTE, 2018):

$\begin{array}{ll}\text { Profesional } & \text { Aprendiz: Mejora la práctica aprendiendo de y con otros, explorando y } \\ \text { empoderado } & \text { probando para apalancar el uso de las TIC en sus estudiantes y mejorar su } \\ & \text { aprendizaje. }\end{array}$

Líder: Busca oportunidades de liderazgo para apoyar el empoderamiento y el éxito de los estudiantes y para mejorar la enseñanza y el aprendizaje.

Ciudadano digital: inspiran a los estudiantes a contribuir positivamente y participar de manera responsable en el mundo digital.

Figura 2. Estándares ISTE para educadores (2018). Elaboración y traducción del autor. 
Colaborador: Dedica tiempo para colaborar con colegas y estudiantes para mejorar la práctica, descubrir y compartir recursos e ideas, y resolver problemas.

Diseñador: diseña actividades y entornos auténticos, impulsados por el alumno; reconoce y adapta según perfil de estudiante.

Facilitador: facilita aprender con tecnología para apoyar los estándares de estudiantes de las Normas ISTE 2016.

Analista: entiende y emplea los datos para orientar su práctica y apoyar a los estudiantes en alcanzar su meta de aprendizaje.

Figura 3. Estándares ISTE para educadores (2018). Elaboración y traducción del autor.

En Colombia ha sido el Ministerio de Educación Nacional - MEN- quien propone las competencias TIC para docentes, como base para desarrollar la innovación educativa. Incluyen lo tecnológico, comunicativo, pedagógico, investigativo y de gestión (figura 4). Designa, además, grados de experticia para cada una de ellas así: Explorador, integrador e innovador (Ministerio de Educación Nacional, 2013).

$\begin{array}{ll}\text { Competencia } & \text { Capacidad para seleccionar y utilizar de forma pertinente, responsable } \\ \text { Tecnológica } & \text { y efi ciente una variedad de herramientas tecnológicas entendiendo } \\ & \text { los principios que las rigen, la forma de combinarlas y las licencias que } \\ & \text { las amparan. }\end{array}$

$\begin{array}{ll}\text { Competencia } & \text { Capacidad para expresarse, establecer contacto y relacionarse en } \\ \text { Comunicativa } & \text { espacios virtuales y audiovisuales a través de diversos medios y con } \\ & \text { el manejo de múltiples lenguajes, de manera sincrónica y asincrónica. }\end{array}$

\begin{tabular}{ll}
\hline Competencia & Capacidad de utilizar las TIC para fortalecer los procesos de \\
Pedagógica & enseñanza y aprendizaje, reconociendo alcances y limitaciones de la \\
& incorporación de estas tecnologías en la formación integral de los \\
& estudiantes y en su propio desarrollo profesional.
\end{tabular}

\begin{tabular}{ll}
$\begin{array}{l}\text { Competencia de } \\
\text { Gestión }\end{array}$ & $\begin{array}{l}\text { Capacidad para utilizar las TIC en la planeación, organización, } \\
\text { administración y evaluación de manera efectiva de los procesos } \\
\text { educativos; tanto a nivel de prácticas pedagógicas como de desarrollo } \\
\text { institucional. }\end{array}$ \\
\hline $\begin{array}{l}\text { Competencia } \\
\text { Investigativa }\end{array}$ & $\begin{array}{l}\text { Capacidad de utilizar las TIC para la transformación del saber y la } \\
\text { generación de nuevos conocimientos. }\end{array}$
\end{tabular}

Figura 4. Competencias TIC para los docentes en el marco de la innovación. Elaboración propia a partir de: Ministerio de Educación Nacional (2013, p 31-33).

En lo que respecta a la Universidad de La Sabana, los profesores deben contar con la denominada Competencia en Informática Educativa- CIE-, definida con la Reglamentación 35 de 2010, donde se estipula como aquella capacidad del profesor para apropiar, integrar e innovar en su práctica docente aplicando las TIC (Universidad de La Sabana, 2010). La misma 
reglamentación establece las condiciones que debe atender el profesor para alcanzar su pleno desarrollo y lo hace fijando unos estándares así:

1 er.

estándar:identifica accede, usa, evalúa información usando TIC.

\section{2o.estándar:} integra TIC para mejorar calidad

enseñanza en

Ambientes

Aprendizaje. 3er. estándar:

Promueve uso

TIC en estudiantes

para proceso de aprendizaje.
DOCENTE COMPETENTE

40. estándar: INNOVA su INNOVADOR

Figura 5. Estándares Competencia Informática Educativa para cuerpo profesoral, Universidad de La Sabana. Elaboración propia a partir de Reglamentación 35 de 2010 (p.6).

La meta, es convertirse en docente competente e innovador (figura 5), estadio donde se es capaz no solo de utilizar las tecnologías y promover su uso, sino de originar productos de conocimiento.

\subsection{Avances en el tema}

Sobre la investigación de las competencias digitales en los profesores universitarios, se encuentran varias tendencias: en primer lugar, quienes se han dedicado a conceptualizar sobre el término (Gisbert Cervera, González Martínez y Esteve Mon, 2016; Durán Cuartero, Gutiérrez Porlán, y Prendes Espinosa, 2016), evolución histórica (Padilla-Hernández, Gámiz Sánchez y Romero-López, 2018), la propuesta de modelos (Hernández, Gamboa y Ayala, 2014; Prendes Espinosa, Gutiérrez Porlán y Martínez Sánchez, 2018) y revisiones documentales sobre el tópico (Rangel Baca, 2018; Zavala, Muñoz y Lozano, 2016; Morales-Pacavita, 2016 ).

En segundo lugar, aparecen estudios que se enfocan en examinar a los profesores, bien desde su propia mirada o la de sus estudiantes; ya sea para establecer el desarrollo de tales competencias (Prendes Espinosa y Gutiérrez Porlán, 2013; Restrepo y Navío 2016; Carrera Farrán y Coiduras Rodríguez, 2012; Hennig Manzuoil y Segovia Cifuentes, 2013), la evaluación de esas habilidades (Durán Cuartero, Gutiérrez Porlán, y Prendes Espinosa, 2016; Vera Noriega, Torres Moran \& Martínez García, 2014), las necesidades formativas en torno a ellas (Pozos Pérez, 2015, Sancho, Ornellas, Sánchez, Alonso y Bosco, 2008; Chou Rodríguez, Valdés Guada y Sánchez Gálvez, 2017; Romero-Martín, Castejón-Oliva, López-Pastor y Fraile-Aranda, 2017) o el perfil competencial del profesor (Salinas lbañez, De Benito y Lizana Carrió, 2014; Rangel Baca, 2015; Cejas y Navío 2016; Ríos Ariza, Gómez Barajas y Rojas Polanco, 2018; Arias Gómez, et al., 2018 ).

No obstante, aunque los estudios mencionados en su gran mayoría se ubican en la educación superior, apenas una mínima porción examina lo que sucede en posgrado, razón de peso para ocuparse ahora del tema.

\section{MÉTODO}

\subsection{Enfoque y diseño del estudio}

El presente estudio desarrollado en el nivel de posgrado opta por un enfoque mixto para neutralizar sesgos de determinados métodos al emplearlos en forma aislada, tal como lo 
describen Rodríguez y Valldeoriola (2010). En esa medida, el procedimiento escogido es concurrente, pues se utilizan de manera simultánea metodologías cualitativas y cuantitativas para lograr una mejor comprensión del objeto de estudio (Creswell, 2009). Se acude a la descripción, dado que la investigación se realiza con una población y un contexto específicos que lo sitúan como un estudio de caso; este diseño, permitirá comprender aspectos característicos de un entorno e individuos que son el centro de interés, a partir de la información que proveen los informantes que conforman la muestra.

\subsection{Población y muestra}

Para el presente artículo, se ha acudido a la población de estudiantes de dos unidades académicas que imparten programas de posgrado en educación: la Facultad de educación con cuatro programas de maestría (uno de ellos virtual) y el Centro de Tecnologías para la Academia, con dos maestrías (una presencial y otra virtual. Los participantes estaban ubicados entre el segundo y último semestre.

La muestra es de tipo No probabilístico, por conveniencia, ya que los sujetos participantes fueron accesibles al investigador y aceptaron participar voluntaria y anónimamente en esta fase del estudio (Otzen y Manterola, 2017). En esa medida, de la población conformada por 470 estudiantes cursando desde segundo hasta último semestre (cuarto o quinto según modalidad del programa de posgrado), de manera voluntaria respondieron, 245 estudiantes, equivalente al $52 \%$.

Un factor a tener en cuenta sobre la población de maestrantes es que está conformada casi en su totalidad (98\%) por profesores adscritos a la Secretaría de Educación Distrital -SED- que han sido becados por el gobierno local para alcanzar la formación como magísteres, y que en su mayoría no han realizado estudios después de obtener el pregrado.

\subsection{Instrumentos}

En lo que respecta al diseño de los instrumentos, se partió de los Estándares de competencias TIC para el profesorado de la Unesco (2008), el MEN (2013) y la Reglamentación 3510 de la Universidad de La Sabana. Adicionalmente, los aportes de Carreras y Coiduras (2012), quienes consideran que además del aula, la calidad de la práctica docente se manifiesta en los siguientes aspectos: planificación, selección y gestión de recursos, regulación y evaluación de los procesos de aprendizaje del estudiantado, entre otros, que orientaron la formulación de las preguntas.
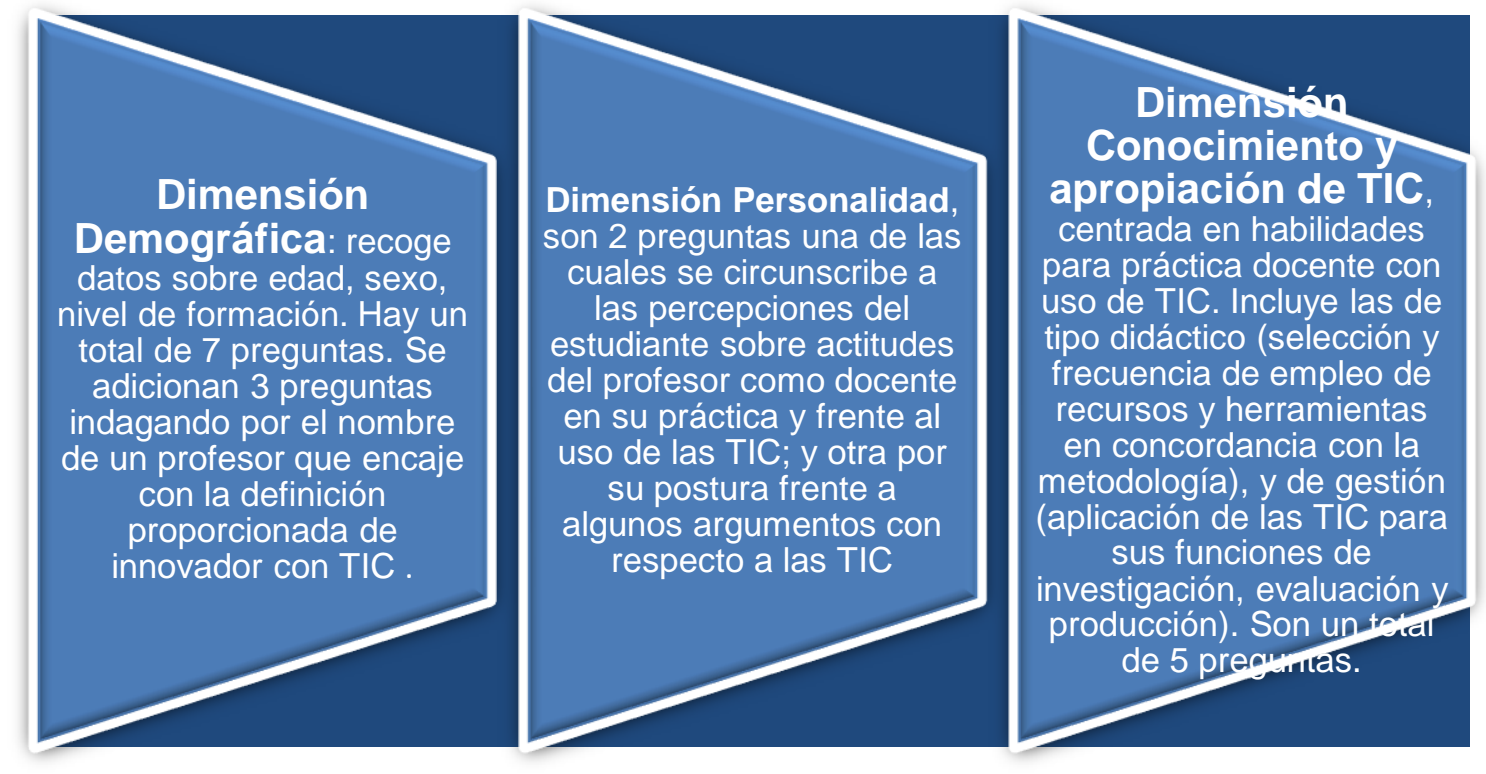

Figura 6. Dimensiones del instrumento. Elaboración propia. 
Así, partiendo de estos autores, se plantearon 3 dimensiones: demográfica, conocimiento y apropiación de TIC y personalidad, bajo las cuales se diseñaron 17 preguntas con un total de 70 variables. El instrumento se sometió a validación de expertos para su posterior ajuste y aplicación (Figura 6).

Prevalecieron las preguntas de selección múltiple y con escala de Likert. El instrumento fue auto administrado y se acudió a la aplicación Google forms, por la facilidad para su envío y diligenciamiento en línea, entre otras ventajas.

\section{RESULTADOS}

\subsection{Dimensión demográfica}

La primera de las dimensiones se centra en describir la muestra de población participante. Para ello, en la tabla 1, se desglosan los hallazgos:

Tabla 1. Caracterización de la muestra

\begin{tabular}{|c|c|c|}
\hline Variables & Estudiantes & Docentes* \\
\hline Edad & $56,5 \%$ por debajo de 40 años & $\begin{array}{l}54.7 \% \text { por encima de los } 41 \\
\text { años }\end{array}$ \\
\hline Género & $65,30 \%$ mujeres & $54,7 \%$ mujeres \\
\hline \multirow[t]{2}{*}{ Áreas de formación } & $\begin{array}{l}61 \% \text { Ciencias de la } \\
\text { educación, } 12 \% \text { Ciencias } \\
\text { sociales y humanas }\end{array}$ & $\begin{array}{l}45 \% \text { Ciencias de la } \\
\text { educación. } 30 \% \text { Ciencias } \\
\text { sociales y humanas }\end{array}$ \\
\hline & $\begin{array}{l}18 \% \quad \text { Ingenierías, } \\
\text { Matemáticas y afines }\end{array}$ & $16 \%$ otros \\
\hline \multirow[t]{2}{*}{ Máximo nivel formación } & $63,2 \%$ Pregrado & $81 \%$ maestría \\
\hline & 35,8\% especialización & $17,2 \%$ doctorado \\
\hline \multirow{2}{*}{$\begin{array}{l}\text { Unidad académica a la que } \\
\text { pertenece }\end{array}$} & 66,6\% Facultad educación & 53\% Facultad educación \\
\hline & $\begin{array}{l}33,4 \% \text { Centro de Tecnologías } \\
\text { para la Academia }\end{array}$ & $\begin{array}{l}\text { 47\% Centro de Tecnologías } \\
\text { para Academia. }\end{array}$ \\
\hline $\begin{array}{l}\text { Modalidad del programa } \\
\text { cursado/orientado }\end{array}$ & $76,3 \%$ presencial & $84,4 \%$ presencial \\
\hline
\end{tabular}

Nota: * se incluye la caracterización de los docentes como complemento, aunque respondiendo al objetivo del presente escrito, no se tratarán sus respuestas.

Un dato de interés que arroja esta primera dimensión es el porcentaje de estudiantes que no identifican profesores que puedan calificarse como innovadores con el uso de TIC (27\%), de los cuales casi la totalidad pertenecen a los programas de la Facultad de educación: 24,5\%.

\subsection{Dimensión Conocimiento y apropiación de TIC}

En primer lugar, se indagó por las habilidades relacionadas con la didáctica y las TIC: selección de estrategias pedagógicas y recursos TIC que empleaban. Se destacaron el Aprendizaje Basado en Problemas -ABP-, desarrollo de proyectos, trabajo colaborativo y trabajo autónomo (figura 7). Los estudiantes observaron que los profesores emplean principalmente los 
foros para el trabajo colaborativo (49\%); lo hacen en menor porcentaje para el trabajo autónomo $(33,5 \%)$, el ABP $(31 \%)$ y el desarrollo de proyectos $(23 \%)$.

El almacenamiento en la nube y los portafolios digitales se utilizan en igual proporción para el desarrollo de proyectos (26\%), y de manera aproximada para el trabajo autónomo (25\% y $22 \%$ respectivamente) y el $A B P(16 \%$ Y $17 \%$ respectivamente). En lo que atañe al trabajo colaborativo los profesores acuden con menor frecuencia a los portafolios (13\%).

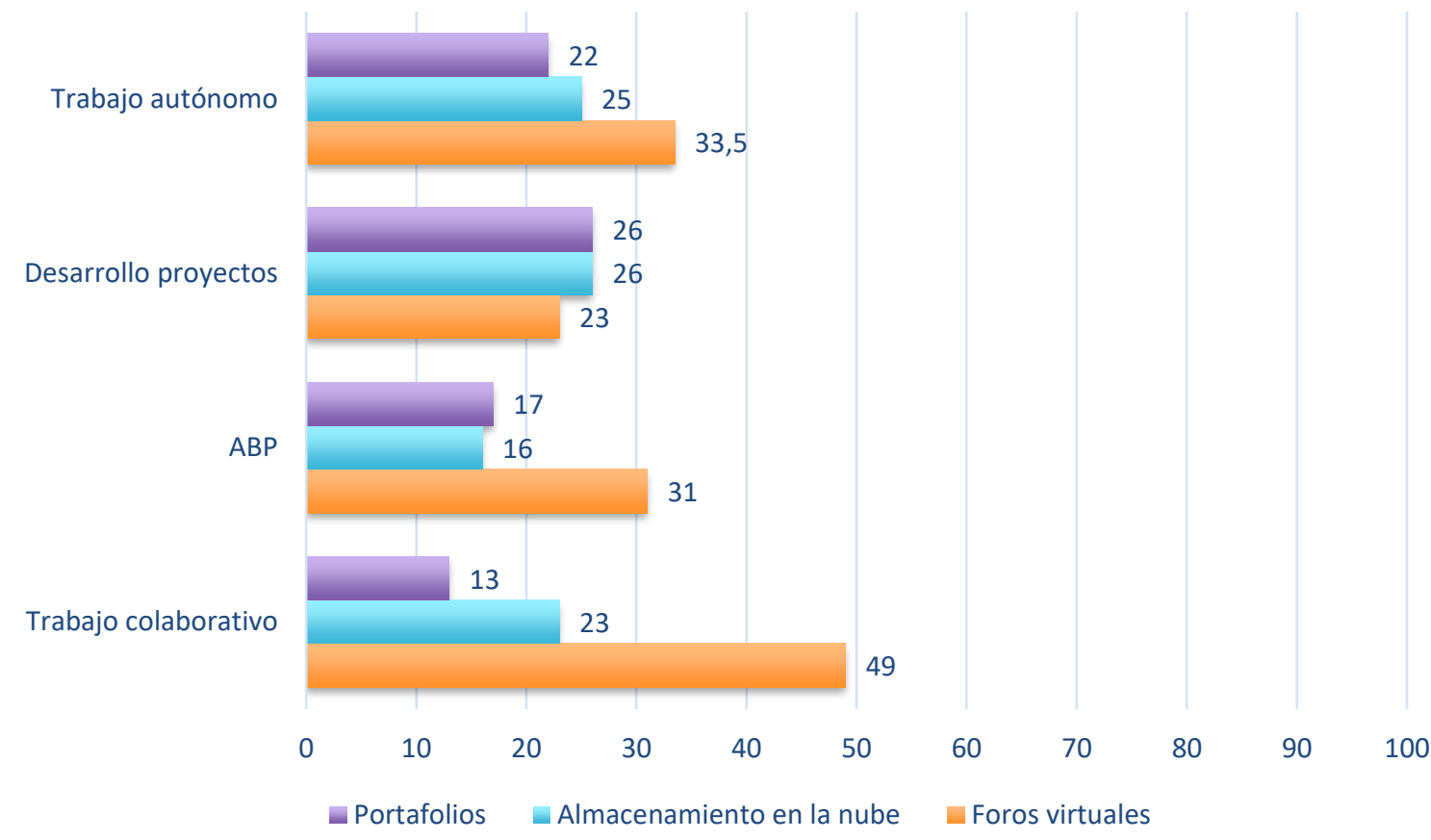

Figura 7. Uso de recursos TIC según estrategias pedagógicas. Fuente: autor.

Asimismo, se examinaron el tipo de recursos que soportan las sesiones de clase/tutoría, en este nivel de formación. En la figura 8 es visible que los estudiantes desde su experiencia afirman que es constante el empleo de las plataformas educativas $(71,5 \%)$, seguido de los videos y multimedia (64\%) y en proporciones similares los motores de búsqueda (55\%), las bases de datos $(52,5 \%)$ y el almacenamiento en la nube $(50 \%)$.

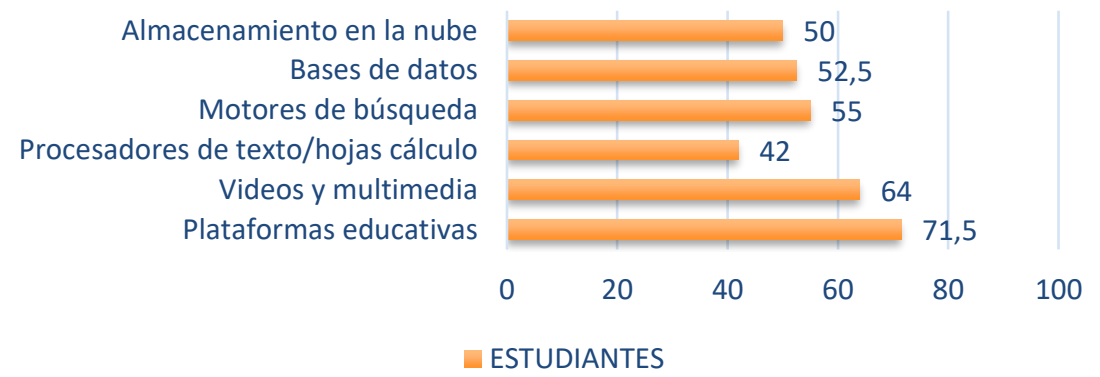

Figura 8. Recursos TIC empleados siempre para clase. Fuente: autor.

Otro tema de interés estuvo relacionado con la gestión de su práctica en asuntos como la investigación y los procesos de evaluación y el tipo de recursos asociados a ellas. Al respecto, los estudiantes sostienen que, para la evaluación, los profesores emplean de manera continua los videos y multimedia (47\%), seguidos por el almacenamiento en la nube $(40 \%)$; en menores pero similares frecuencias acuden a los mapas mentales (38\%) procesadores de texto $(37 \%)$ y portafolios (34\%), concediendo a estos tres un empleo ocasional (Figura 9). 


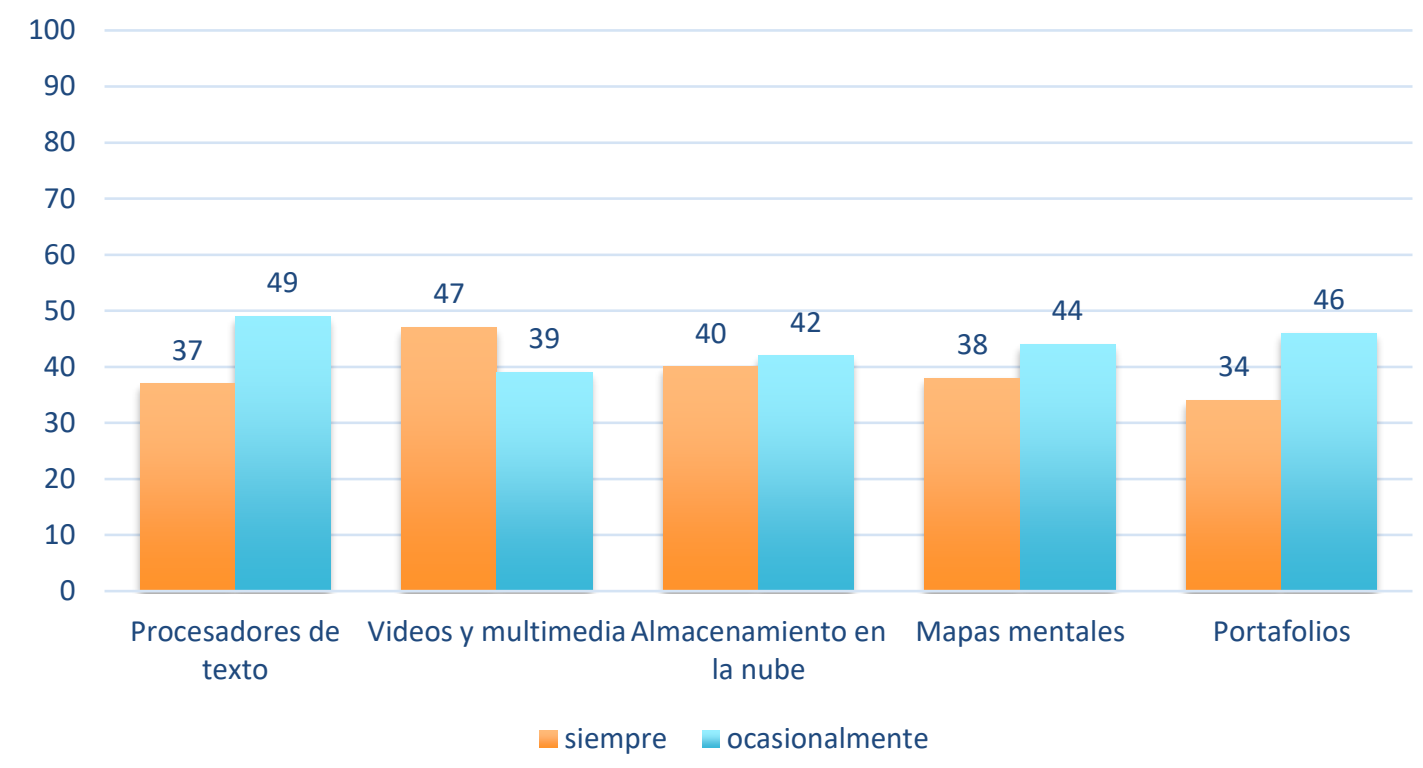

Figura 9 . Recursos TIC empleados siempre y ocasionalmente para evaluación. Fuente: autor

En síntesis, esta población considera que el empleo de recursos TIC no presenta mayor regularidad. Algunos recursos no se utilizan tal como se observa en la figura 10.

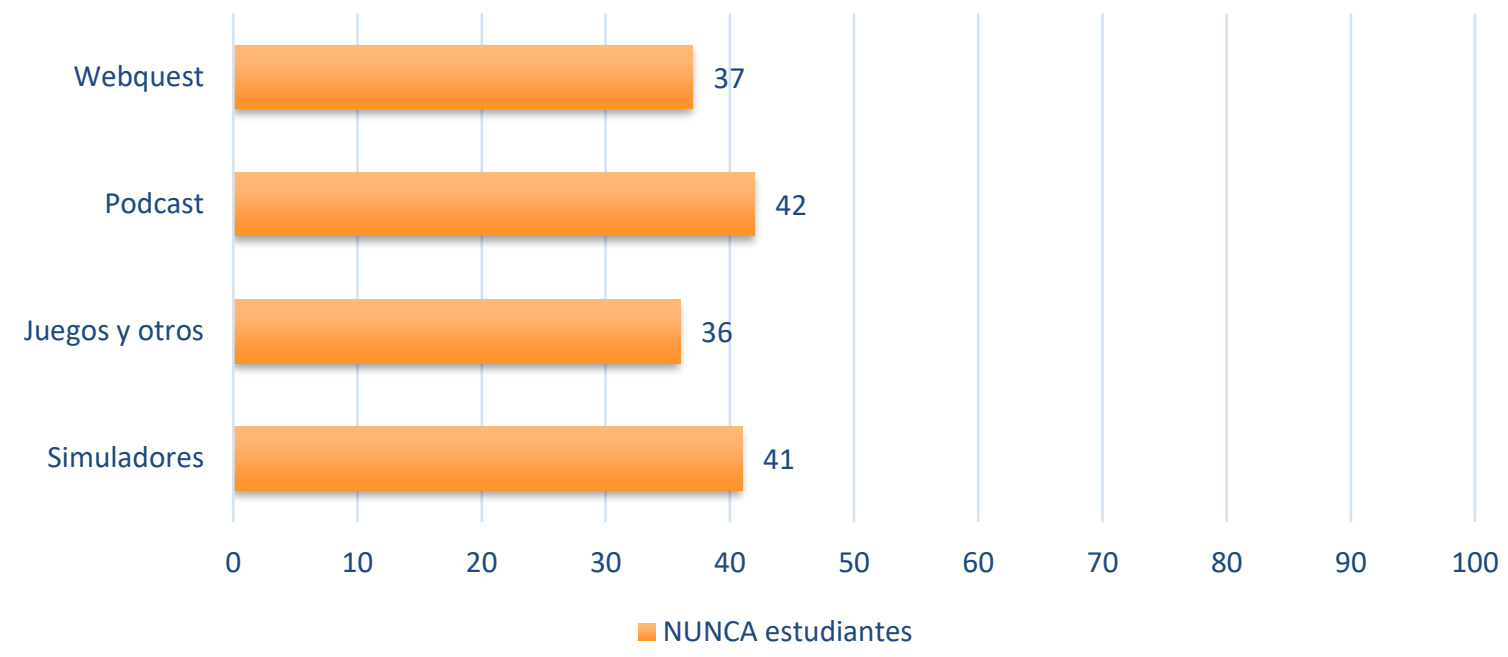

Figura 10 Recursos TIC que nunca se emplean en Evaluación. Fuente: autor.

Durante la aplicación del instrumento, fue manifiesto el desconocimiento de tales herramientas de parte de los maestrantes, donde buena parte solicitó explicación de manera recurrente sobre los términos empleados.

En cuanto a la actividad de investigación (figura 11), los estudiantes hacen de nuevo hincapié en el empleo de plataformas educativas $(60 \%)$ y le siguen los motores de búsqueda (54\%) y los videos y material multimedia (52,5\%). También acuden a las bases de datos $(49,7 \%)$, el almacenamiento en la nube y los procesadores de texto (45,8\% ambos). 


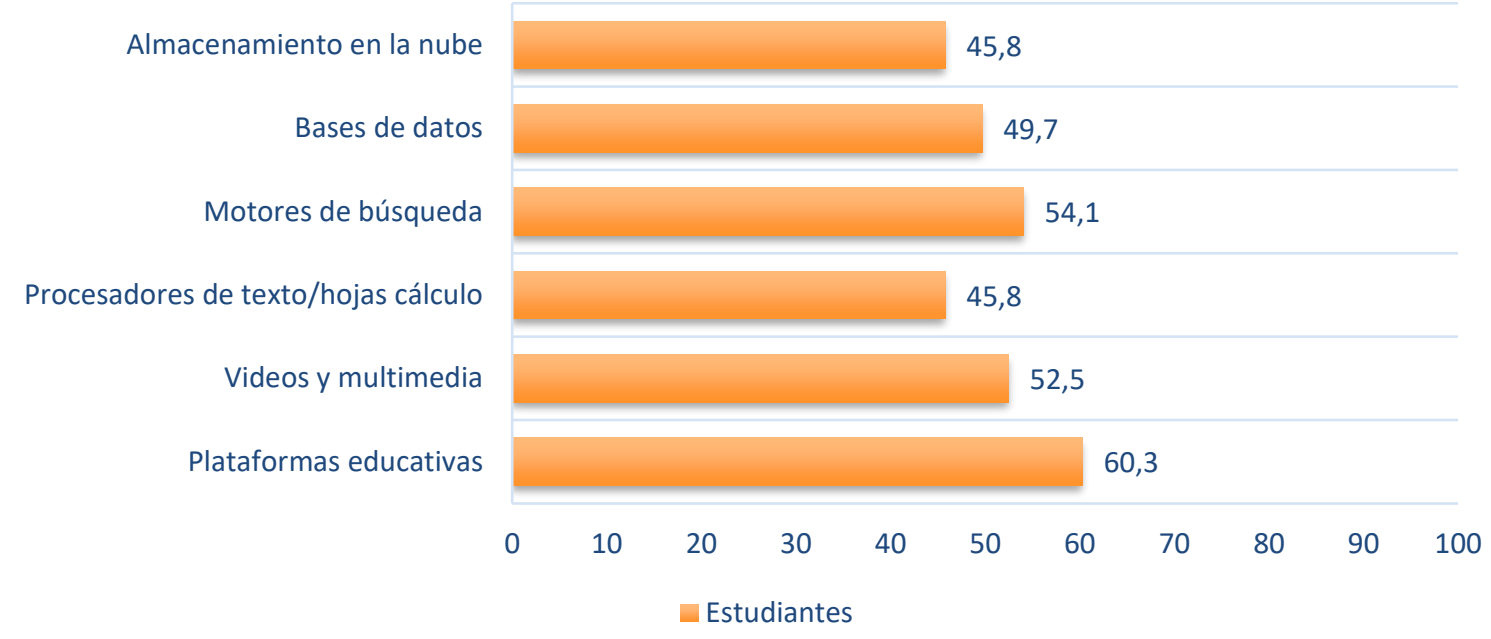

Figura 11. Recursos TIC empleados frecuentemente para investigación. Fuente: autor.

Es de anotar que, según los maestrantes y con una periodicidad ocasional de empleo, emergen recursos que no han sido contemplados previamente como el caso del software especializado (42\%) y los gestores bibliográficos (43\%) (figura 12).

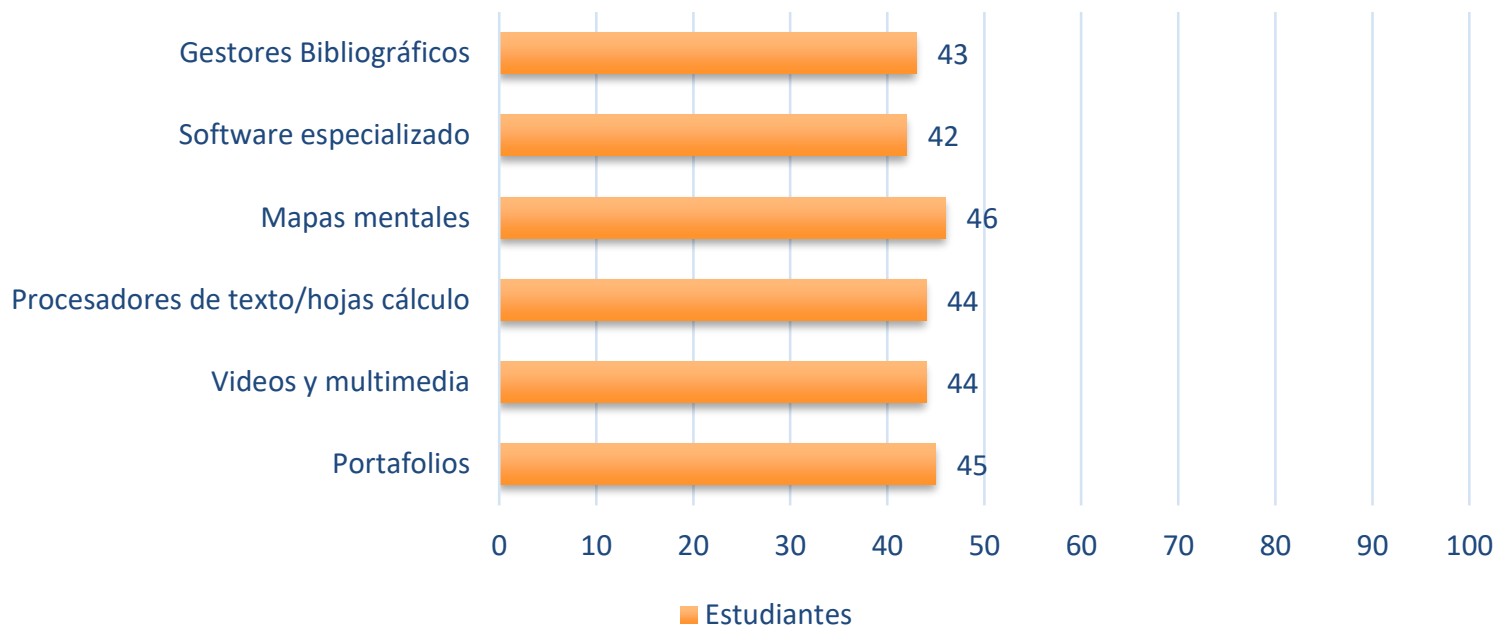

Figura 12. Recursos TIC empleados ocasionalmente para investigación. Fuente: autor.

Como se ha descrito, en esta dimensión se buscó constatar cómo se asumen las TIC en la práctica y para ello se realizaron indagaciones que atañen al ejercicio del profesor en torno a trabajo colaborativo, diseño y renovación de material, socialización de producción y pertenencia a redes profesionales (figura 13) entre otras. Cada una de estas actividades implica hacer uso de las TIC o se hace necesario acudir a ellas para mayor eficiencia. Este ítem también permite establecer si los profesores hacen explícitas en su acción estas actividades, que a la postre van encaminadas a una población caracterizada por prácticas tradicionales en donde las TIC no ocupan un lugar preferente.

Los resultados arrojan que los estudiantes reconocen que de manera continua sus profesores renuevan material (84\%), diseñan material multimedia (81\%), trabajan colaborativamente $(79 \%)$, elaboran documentos $(76 \%)$, pertenecen a redes profesionales y académicas $(74 \%)$ y socializan su producción $(71 \%)$. En menor proporción admiten que posean blogs $(45 \%)$ y páginas web (35\%). 
Sobre estos dos últimos recursos se resalta, que un porcentaje cercano a la mitad de la población de estudiantes desconoce su existencia en propiedad de los docentes (43\% blog y $53 \%$ página web).

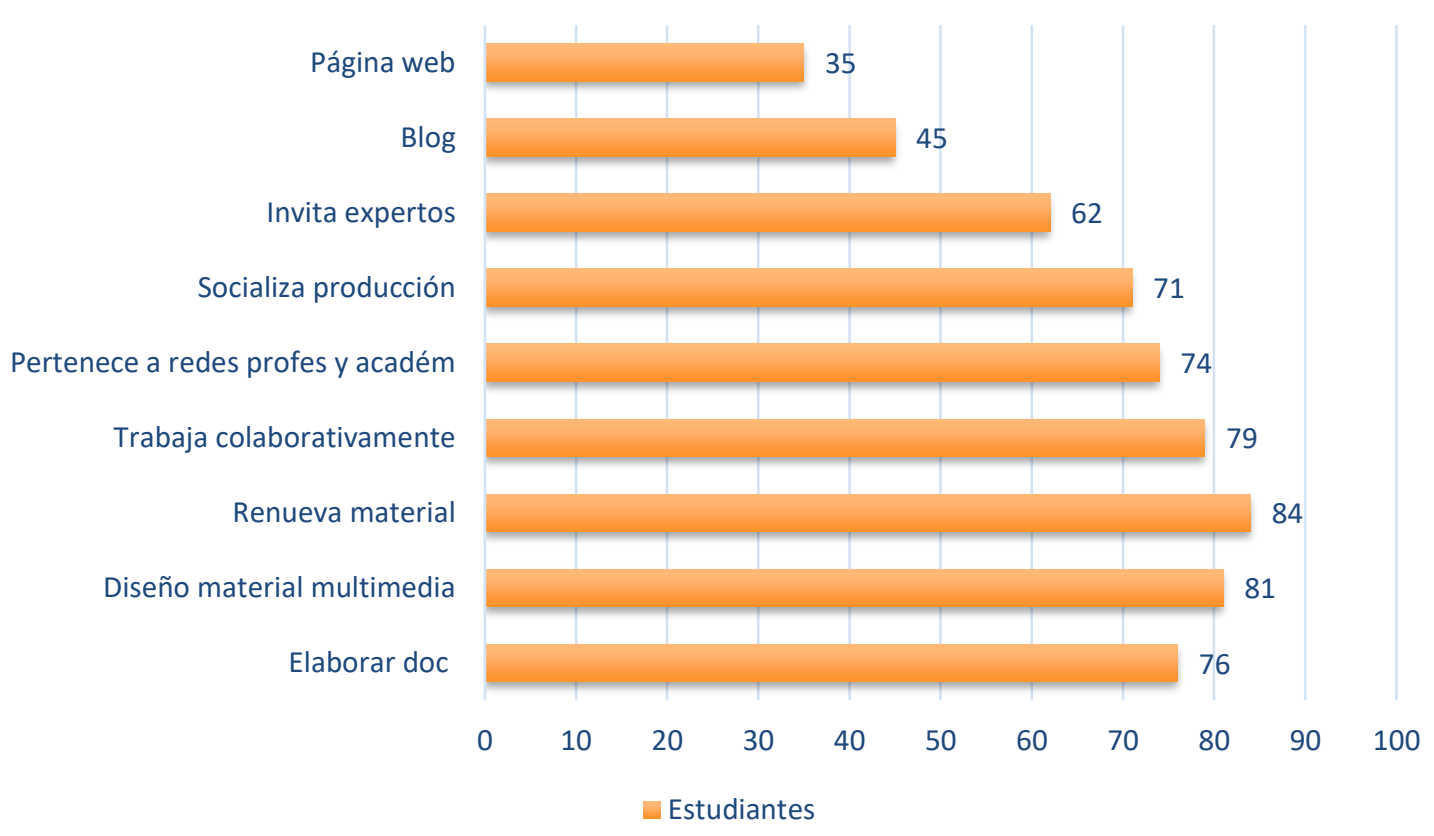

Figura 13. Actividades que evidencian apropiación y conocimiento de TIC. Fuente: autor.

\subsection{Dimensión personalidad}

En esta última dimensión, se indaga sobre la importancia que conceden los profesores a ciertos procesos durante su interacción con el estudiantado. Se han tomado para el análisis aquellas respuestas ponderadas con 5 (figura 14).

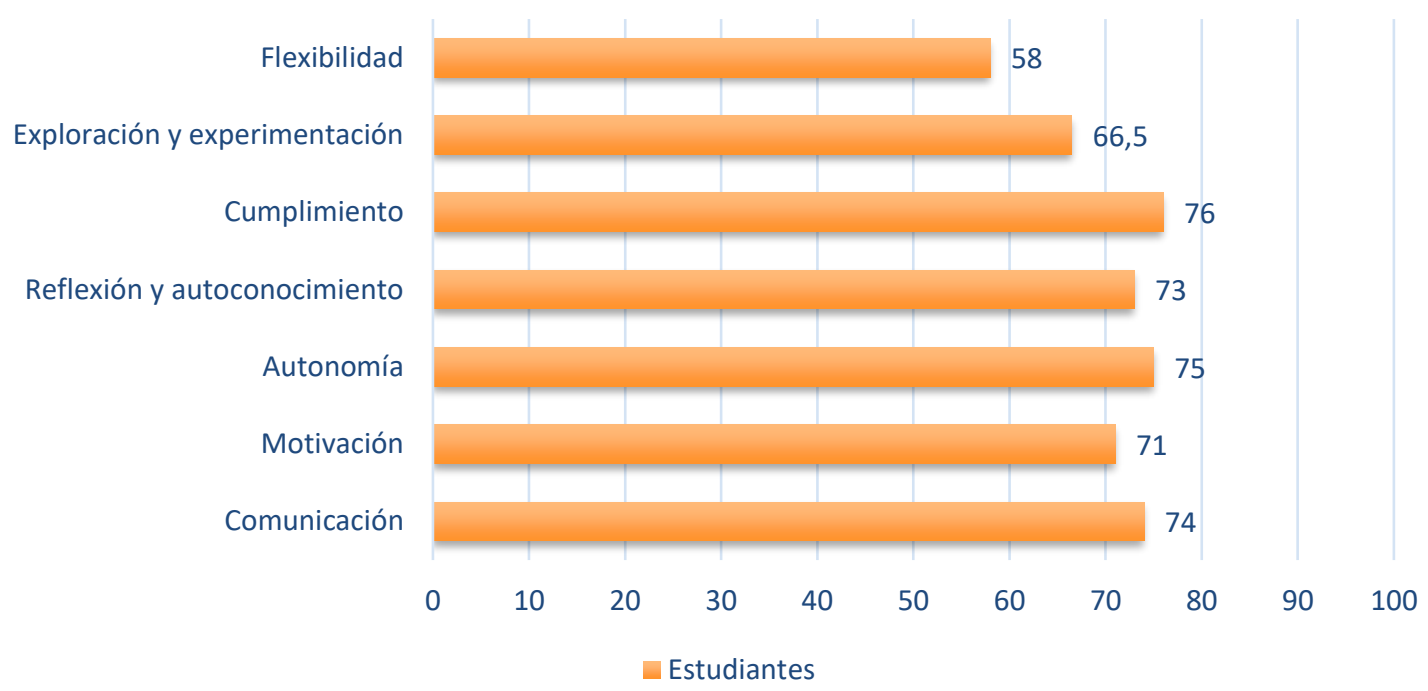

Figura 14. Importancia de algunos procesos en la interacción de clase. Fuente: autor

Según los estudiantes, sus profesores conceden la mayor importancia al cumplimiento (76\%), seguido de cerca por la autonomía (75\%), la comunicación $(74 \%)$, la reflexión y autoconocimiento $(73 \%)$ y la motivación $(71 \%)$. La exploración y la flexibilidad aparecen como aquellos procesos que menos favorecen los profesores. 


\section{DISCUSIÓN Y CONCLUSIONES}

La dimensión sobre conocimiento y apropiación de TIC deja en claro que, según los maestrantes, las plataformas son una herramienta de frecuente utilización por los profesores. Este hecho, puede asociarse con la disponibilidad de la plataforma institucional Virtual Sabana, para apoyar diversas actividades, particularmente en programas en modalidad virtual. Se revela así, que los profesores conocen, manejan y se apropian de los recursos que la institución pone al alcance, logrando de manera colateral la alfabetización de sus estudiantes, quienes mayoritariamente laboran en instituciones educativas del Estado, cuya infraestructura tecnológica no siempre está actualizada.

A la par de las plataformas, aparecen favorecidos con su frecuencia de empleo, los videos y multimedia, cuya función no solo es apoyar las clases, sino procesos como la evaluación. Adicionalmente, para las actividades de investigación los profesores acuden a los motores de búsqueda, las bases de datos y el almacenamiento en la nube, todas ellas pertinentes a ese propósito, tal como han afirmado algunos autores al considerar las TIC como fundamentales para hacer eficaz cualquier proceso de búsqueda y manejo de información (Wilson, Grizzle, Tuazon, Akyempong \& Cheung, 2011).

Se observa también, congruencia en la selección de los recursos TIC con respecto al tipo de estrategia pedagógica a emplear; si se trata de trabajar con otros como el caso del trabajo colaborativo o el ABP, los medios deben corresponder a esos propósitos y en esa medida son pertinentes los foros. Asimismo, concuerdan los portafolios como soporte para el trabajo autónomo y el desarrollo de proyectos, tal como se ratifica en las respuestas.

No obstante, es limitada la variedad de recursos a los que se acude en la práctica. El uso ocasional de portafolios, software especializado, gestores bibliográficos o mapas mentales, dan cuenta de ello. Además, es inobjetable que se prescinde de nuevas herramientas como el caso de los simuladores, juegos y otros, lo que sorprende en programas de formación asociados a la educación, en donde tienen gran aplicación. Se desprende de ahí, que hay conformidad con el empleo de herramientas básicas y de uso generalizado como los procesadores de texto, los foros, los videos y multimedia, ignorando la amplia gama que de forma gratuita hoy en día ofrece la web y más aún, otras posibilidades que ofrece la institución que cuenta con una robusta infraestructura tecnológica.

Aunado a lo anterior, la experiencia de aplicación del instrumento dio lugar a identificar que, para una parte de la población perteneciente a los programas de la facultad de Educación, algunos de los términos o denominaciones técnicas de los recursos digitales les eran desconocidos, a pesar de identificarlos cuando se les explicaba. Otro asunto, es que los profesores pasan por alto socializar sus publicaciones o producción con el alumnado, que tampoco puede dar cuenta de la creación o participación en blogs y páginas web de sus formadores. Esto no solo atañe a la dimensión de conocimiento y apropiación de TIC, sino a la misma Reglamentación 35 de la universidad, que demanda como evidencia de la CIE, la transformación del conocimiento en productos digitales (Universidad de La Sabana, 2010).

A partir de las anteriores afirmaciones, es posible situar a los profesores que fueron seleccionados por sus estudiantes como innovadores, en el nivel de integrador para las competencias tecnológica, comunicativa y pedagógica, tal como lo describe el Ministerio de Educación Nacional (2013) en sus estándares. Esto obedece, a que utilizan las TIC para el apoyo del proceso en el aula aprovechando su potencial pedagógico y también permitiendo gestionar la información; del mismo modo, se valen de ellas para el trabajo autónomo y como herramienta asincrónica en la virtualidad, todos ellos relacionados con el grado de apropiación de las TIC en los docentes.

Atendiendo al ISTE y sus estándares 2018, no se observa que cumplan con todo lo previsto para considerarse un profesional empoderado de las tecnologías. Los roles de líder, aprendiz o ciudadano digital se dibujan como incipientes ante la ausencia de la exploración o el carácter de inspirador. Igual sucede con su papel de impulsor de aprendizaje con TIC, en donde solo es notorio su interés por el diseño de actividades y particularmente en la renovación del material. 
De otro lado y basados en las competencias que exige la institución a su planta de profesores, a partir de estos hallazgos es notable que se ubican en el segundo estándar de la $\mathrm{CIE}$, por cuanto se limitan a incorporar las TIC como apoyo a su práctica, distando mucho de lo que se espera en los últimos estándares donde se espera que promueva el uso de las TIC, innove en su práctica y se ubique como docente competente e innovador (Universidad de La Sabana, 2010).

Sobre la última dimensión inherente a la personalidad del educador, aparecen el cumplimiento, la autonomía y la comunicación, como factores que los estudiantes señalan de mayor importancia para sus docentes. Esto corresponde con lo que se espera en términos de formación de una institución acreditada y reconocida a nivel nacional por el CNA y que coloca como centro a la persona. Se espera, que los profesores con exigencias de esta índole se constituyan en ejemplo para aquellos que se desempeñan como educadores. Además, y atendiendo a las afirmaciones de Cejas y Navío (2016), la actuación del profesorado universitario a partir de la mediación de las TIC, debe ofrecer nuevas posibilidades al estudiantado creando entornos enriquecidos que propicien simultáneamente la colaboración y la autonomía entre otros.

Desde su experiencia, los estudiantes han manifestado que la competencia digital de los profesores tal como se describe en el postulado de Suárez-Rodríguez, Almerich, Díaz-García, y Fernández-Piqueras (2012), no se encuentra plenamente desarrollada. No se identifica una plena integración de las TIC a la práctica y el tipo de recursos como ya se ha mencionado es limitado. De otro lado y examinando los estándares que soportan este escrito, es notorio que debe promoverse la creación de productos de conocimiento que puedan ser aprovechados por los estudiantes.

Surge el cuestionamiento, además, sobre los programas de educación, donde se identificaron vacíos de conocimiento de la terminología relacionada con las TIC, y figura el mayor porcentaje de población que desconoce profesores innovadores que empleen las tecnologías. Esta situación invita a revisar cómo se encuentra la competencia digital de los profesores a cargo de formar maestros, en un espacio particularmente propicio para la actualización.

Finalmente, entre las limitaciones del estudio se encuentra la restringida posibilidad de acceso a las poblaciones en cuestión, dado sus limitados tiempos en la institución ceñidos al fin de semana y con cronogramas muy ajustados. Asimismo, la respuesta a los cuestionarios en línea está sujeta a la buena voluntad y disposición de los maestrantes principalmente en los programas virtuales, pues no hay otra forma de comunicación con ellos. Este proceso de recogida de información dilata y afecta los tiempos establecidos inicialmente en el cronograma.

Sobre futuras investigaciones, es importante ahora confrontar las percepciones de ambos actores, para determinar cuál es la realidad de la práctica que involucra las TIC en este nivel de formación posgradual. También sería prioritario ocuparse de establecer cuál es el nivel de competencia tecnológica en que se encuentra este profesor que asume la formación de profesionales en el campo de la educación.

\section{ENLACES}

Enlace a los instrumentos de recogida de datos: https://goo.gl/forms/cx2LNpjEbWMvXpJ32

\section{REFERENCIAS BIBLIOGRÁFICAS}

Arias Gómez, M. de L., Arias Gómez, E., Arias Gómez, J., Ortíz Molina, M. M. y Garza García, M. G. del C. (2018). Perfil y Competencias del docente universitario recomendados por la UNESCO y la OCDE. Atlante: Cuadernos de Educación Y Desarrollo, 1-21. Recuperado de https://www.eumed.net/rev/atlante/2018/06/competencias-docente-universitario.html 
Cejas, R., Navío, A. y Barroso, J. (2016). Las competencias del profesorado universitario desde el modelo TPACK (Conocimiento Tecnológico y Pedagógico Del Contenido). Píxel-Bit. Revista de Medios y Educación, 49, 105-119. doi: 10.12795/PIXELBIT

Cejas, R. y Navío, A. (2016). Las competencias del profesorado universitario que asiste a formaciones TIC. El caso de la Universitat Autònoma de Barcelona. Recuperado de https://www.researchgate.net/publication/312951056\%0

Carrera Farrán, F. X. y Coiduras Rodríguez, J. L. (2012). Identificación de la competencia digital del profesor universitario: un estudio exploratorio en el ámbito de las Ciencias Sociales. Revista De Docencia Universitaria, 10(2), 273-298.

Chou Rodríguez, R., Valdés Guada, A. y Sánchez Gálvez, S. (2017). Programa de formación de competencias digitales en docentes universitarios. Revista Universidad Y Sociedad, 9(1), 8186. Recuperado de http://rus.ucf.edu.cu/index.php/rus/article/viewFile/530/587\#page=81

Consejo Nacional de Acreditación - CNA (2010). Lineamientos para la Acreditación de Alta Calidad de Programas de Maestría y Doctorado. Bogotá: Ministerio de Educación Nacional.

Creswell, J.W. (2009). Research Design; qualitative, quantitative and mixed methods approaches. 3a. Ed. Londres: Sage.

Durán Cuartero, M., Gutiérrez Porlán, I. y Prendes Espinosa, M. P. (2016). Análisis conceptual de modelos de competencia digital del profesorado universitario. Revista Latinoamericana de Tecnología Educativa, 15(1), 97-114. doi: 10.17398/1695

Durán Cuartero, M., Gutiérrez Porlán, I. y Prendes Espinosa, M. P. (2016). Diseño y validación de un instrumento de evaluación para la certificación de la competencia TIC del profesorado universitario. Revista Mexicana de Investigación Educativa, 21(69), 527-556. doi: $\underline{10.13140 / 2.1 .4232 .4165}$

Gisbert Cervera, M., González Martínez, J. y Esteve Mon, F. M. (2016). Competencia digital y competencia digital docente: una panorámica sobre el estado de la cuestión. Revista Interuniversitaria de Investigación En Tecnología Educativa- RIITE, (0), 74-83. doi: $\underline{10.6018 / \text { riite2016/257631 }}$

Hennig Manzuoli, C. y Segovia Cifuentes, Y. (2013). Computing Education Competence in Higher Education: Challenges for Teachers. American Journal of Educational Research, 1(9), 406412. doi: 10.12691/education-1-9-9

Hernández, C. A., Gamboa, A. A. y Ayala, E. T. (2014). Competencias para los docentes de Educación Superior. En Congreso Iberoamericano de Ciencia, Tecnología, Innovación y Educación COMPETENCIAS (p. 20). Buenos Aires.

Indrasiene, V., Dromantiene, L. y Bielskyte-Simanaviciene, E. (2015). Use Of Information And Communication Technology In The Study Process: Teachers'experience. Socialines Technologijos, 5(1), 7-21.

International Society for Technology in Education ISTE (2018). ISTE Standards for educators. Recuperado de http://iste.org/standards-

Ministerio De Educación Nacional (2013). Competencias TIC para el desarrollo Profesional Docente. Recuperado de: http://www.colombiaaprende.edu.co/html/micrositios/1752/articles$\underline{318264 \text { recurso tic.pdf. }}$ 
Ministerio De Educación Nacional (2013). Secretaría de Educación de Bogotá invertirá \$35.000 millones para formación docente en 2013. Recuperado de: https://www.mineducacion.gov.co/cvn/1665/w3-article-321209.html

Morales-Pacavita, O. (2016). Desarrollo de competencias tecnológicas en docentes. Una mirada desde la Andragogía. Quaestiones Disputatae-Temas En Debate, 9(18), 229-250.

Otzen, T. y Manterola, C. (2017). Técnicas de Muestreo sobre una Población a Estudio. International Journal of Morphology, 35(1), 227-232. doi: 10.4067/S0717$\underline{95022017000100037}$

Padilla-Hernández, A. L., Gámiz-Sánchez, V. M. y Romero-López, M. A. (2018). Selección de categorías para el estudio de la evolución de la competencia digital docente del profesorado en Educación Superior. RIITE. Revista Interuniversitaria de Investigación en Tecnología Educativa, 4, 55-67. doi: 10.6018/riite/2018/327881

Prendes Espinosa, M. P., Castañeda, L. y Gutiérrez, I. (2010). Competencias para el uso de TIC de los futuros maestros. Comunicar, 17(35), 175-181. doi: 10.3916/C35-2010-03-11

Prendes Espinosa, M. P. y Gutiérrez Porlán, I. (2013). Competencias tecnológicas del profesorado en las universidades españolas. Revista de Educación, 361, 196-222. doi: 10.4438/1988-592X-RE-2011-361-140.

Prendes Espinosa, M. P., Gutiérrez Porlán, I. y Martínez-Sánchez, F. (2018). Competencia digital: una necesidad del profesorado universitario en el siglo XXI. Revista de Educación a Distancia (RED), 56(7), 31-1. doi: 10.6018/red/56/7

Pozos Pérez, K. V. (2016). Evaluación de necesidades de formación continua en competencia digital del profesorado universitario mexicano para la sociedad del conocimiento (Tesis Doctoral). Universidad Autónoma de Barcelona. Recuperada de http://www.tdx.cat/handle/10803/382466

Rangel Baca, A. (2015). Competencias docentes digitales: propuesta de un perfil. Pixel-Bit: Revista de Medios Y Educación, 46(Enero), 235-248. doi: 10.12795/pixebil.2015.i46.15

Rangel Baca, A. (2018). Tecnologías De La Información Y La Comunicación En La Educación Superior: Una Revisión Documental Information and Communication Technologies in Higher Education : a Documentary Analysis. Pixel-Bit. Revista de Medios y Educación, 52, 125-137.

Restrepo, J. (2016). Las competencias del docente de posgrado: una perspectiva desde la formación de programas de maestría (Tesis Doctoral). Universidad Autónoma de Barcelona. Recuperada de: $\underline{\text { https://ddd.uab.cat/record/168476 }}$

Restrepo, J. y Navío, A. (2016). Las competencias del docente de maestría en universidades colombianas: apreciaciones de alumnos y profesores. Civilizar, 16(31), 191-204.

Ríos Ariza, J. M., Gómez Barajas, E. R. y Rojas Polanco, M. P. (2018). Valoración De Competencias Tic Del Profesorado Universitario: Un Caso En Chile. Pixel-Bit, Revista de Medios y Educación, 52, 55-65.

Roa, A. y Pacheco, I.F. (2014). Educación Superior en Colombia. Medellín: Universidad del Norte.

Rodríguez-Beneroso, M. (2014). Análisis y evaluación de la docencia universitaria mediada con tecnologías (Tesis Doctoral). Universidad Autónoma de Barcelona. Recuperada de http://ddd.uab.cat/pub/tesis/2014/hdl 10803 286076/mrb1de1.pdf 
Rodríguez, D. y Valldeoriola, J. (2010). Metodología de la investigación. Universitat Oberta de Catalunya, 613. Recuperado de http://myuvmcollege.com/uploads/lectura201109/Metodolog\%C3\%ADa\%20de\%20investigaci\%C3\%B3n-2064.pdf

Romero-Martín, R., Castejón-Oliva, F. J., López-Pastor, V. M. y Fraile-Aranda, A. (2017). Evaluación formativa, competencias comunicativas y TIC en la formación del profesorado. Comunicar, 52(3). doi: 10.3916/C52-2017-07

Salinas Ibañez, J., De Benito, B. y Lizana Carrió, A. (2014). Competencias docentes para los nuevos escenarios de aprendizaje. Recuperado de http://www.redalyc.org/pdf/274/27431190010.pdf

Sánchez, A., Boix, L. y Jurado, P. (2009). La Sociedad Del Conocimiento y Las Tics: Una Inmejorable Oportunidad Para El Cambio Docente. Revista de Medios Y Educación, 34, 179204.

Sancho, J. M., Ornellas, A., Sánchez Valero, J.A., Alonso, C. y Bosco, A. (2008). La formación del profesorado en el uso educativo de las TIC: una aproximación desde la política educativa. Praxis Educativa, 12(12), 10-22. Recuperado de https://cerac.unlpam.edu.ar/index.php/praxis/article/view/421/351

Suárez-Rodríguez, J., Almerich, G., Díaz-García, I. y Fernández-Piqueras, R. (2012). Competencias del profesorado en las TIC. Influencia de factores personales y contextuales. Universitas Psychologica, 11(1), 293-309.

Tünnerman, C. y De Souza, M. (2003). Desafíos de la universidad en la sociedad del conocimiento. En Cinco años después de la Conferencia Mundial sobre Educación Superior.

Unesco (2008). ICT Competency Standards for Teachers. Implementation Guidelines. Version 1.0. United Nations Educational, Scientific and Cultural Organization.

Universidad de La Sabana (2010). Reglamentación No. 35 de 2010.

Valerio-Ureña, G. y Rodríguez-Martínez, M. del C. (2017). Perfil del profesor universitario desde la perspectiva del estudiante. Innovación Educativa, 17(74), 109-124. Recuperado de http://www.scielo.org.mx/pdf/ie/v17n74/1665-2673-ie-17-74-00109.pdf

Vera Noriega, J. A., Torres Moran, L. E. y Martínez García, E. E. (2014). Evaluación de competencias básicas en TIC en docentes de educación superior en México. Pixel-Bit: Revista de Medios Y Educación, (44), 143-155. doi: 10.12795/pixelbit.2014.i44.10

Wilson, C.; Grizzle, A.; Tuazon, R.; Akyempong, K. y Cheung, C.K. (2011). Alfabetización mediática e informacional: curriculum para profesores. Unesco. doi: 978-92-3-104198-3

Zavala, D., Muñoz, C. y Lozano, E. (2016). Las competencias digitales de los docentes. Publicando, 3(9), 330-340. Recuperado de http://www.rmlconsultores.com/revista/index.php/crv/article/viewFile/353/pdf 219 


\section{INFORMACIÓN SOBRE LOS AUTORES}

\section{Monica Marcela Sánchez Duarte \\ Universidad de La Sabana}

Jefe Recursos pedagógicos programa Core Curriculum Persona \& Cultura. Doctoranda en educación, Magíster en educación, Especialista en gestión del diseño y Diseñadora Industrial. Experiencia de 15 años en la administración y gestión de programas de pregrado en diseño, conduciendo los respectivos procesos de acreditación. En el campo de investigación sobre las TIC en educación superior suman dos décadas, que incluyen dos proyectos financiados por el Departamento Administrativo de Ciencia, Tecnología e Innovación- COLCIENCIAS.

Blog: http://ecoprofesionales.blogspot.com.co/

\section{Antoni Navío Gámez}

Universidad Autónoma de Barcelona

Coordinador del grado en Pedagogía. Profesor de didáctica y organización educativa del Departamento de Pedagogía aplicada. Doctor en educación, Magíster e formación de formadores y Especialista en organización y gestión de centros educativos con formación de grado en Licenciatura en filosofía y letras. La línea de investigación se centra en la formación de formadores.

Los textos publicados en esta revista están sujetos a una licencia de Reconocimiento 4.0 España de Creative Commons. Puede copiarlos, distribuirlos, comunicarlos públicamente y hacer obras derivadas siempre que reconozca los créditos de las obras (autoría, nombre de la revista, institución editora) de la manera especificada por los autores o por la revista. La licencia completa se puede consultar en:Licencia Creative Commons Atribución-NoComercial-Compartir por igual 4.0 Internacional. 